\title{
Photosynthesis and artificial photosynthesis research
}

\author{
Mamoru Nango • Miwa Sugiura
}

Published online: 15 October 2014

(C) Springer Science+Business Media Dordrecht 2014

Since the emergence of modern science, photosynthesis has been the focus of interest of many researchers because of its importance for all forms of life on this planet. In particular, remarkable progress has been made during the last two decades. Of special importance is the determination of the three-dimensional structures of key proteins in photosynthesis, such as Photosystem I, Photosystem II, and cytochrome $b_{6} f$ complexes by $\mathrm{X}$-ray crystallography. These crystallographic studies provide useful information about the static structures of these biomolecules. On the other hand, the dynamic aspects of these biomolecules are equally important, which have been revealed by various other techniques such as biochemical assays, fluorescence spectroscopy, magnetic resonance studies, and so on. These successful research activities have collected an impressive amount of scientific knowledge, although there is still much debate on various important aspects, which are under active research right now.

From the viewpoint of chemistry, the situation looks somewhat different. Although we may be able to understand the molecular system of natural photosynthesis, we are far from being able to re-construct it in our hands. The latter challenge is still too complex for today's chemists. Nevertheless, building up artificial photosynthetic systems on the basis of our knowledge about natural photosynthesis is a worthwhile goal.

\footnotetext{
M. Nango ( $($ )

The Osaka City University Advanced Research Institute for Natural Science and Technology (OCARINA), Sugimoto, Sumiyoshi-ku, Osaka 558-8585, Japan e-mail: nango@sci.osaka-cu.ac.jp

M. Sugiura

Proteo-Science Research Center, Ehime University, Bunkyo-cho, Matsuyama 790-8577, Japan e-mail: miwa.sugiura@ehime-u.ac.jp
} 
This special issue of "Photosynthesis and Artificial Photosynthesis Research" in Research on Chemical Intermediates highlights recent advances of our understanding on photosynthesis summarized the characteristics of the natural system, of applied researches utilized protein complexes relating to the photosynthesis, and of artificial photosynthesis. This issue contains 11 invited and selected papers on photosynthesis research, presented at the international conference "The 70th Okazaki International Conference on Molecular Mechanism of Photosynthetic Energy Conversion: the Present Research and Future Prospects", that was held in Okazaki, Japan, during 4th-6th, December, 2010, with the sponsorship of Institute for Molecular Science, National Institutes of Natural Sciences. There were 108 participants from ten countries. In addition, we had 25 invited speakers and 27 posters. The purpose of this conference was to provide a forum where researchers from various fields related to photosynthesis came together and exchanged their idea. Hot debates on topics at the forefront of each field were successfully done in spite of researchers came from different fields, and we could eventually lead to the emergence of a new research community for photosynthetic energy conversion. Since this conference, we saw much need for publishing a special issue regarding photosynthesis and artificial photosynthesis research.

\section{Memory of Warwick Hillier}

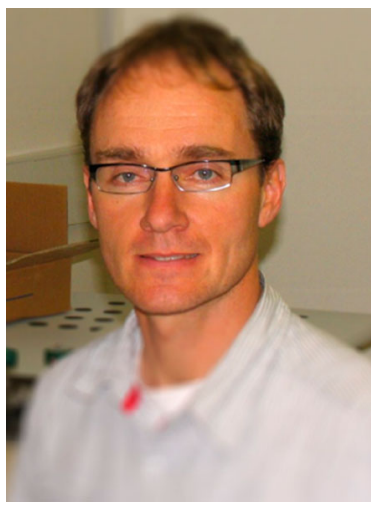

In the conference, Professor Warwick Hillier (Research School of Biology, The Australian National University) gave a talk which was titled "Developments in Stable Isotope Mass Spectrometry: Chemical Insights into Catalysis of Water Oxidation" in the session of "New Approaches for Photosynthesis Research" in the symposium. Just after this conference, he knew that a brain had tumor invaded him. Unfortunately, he went on his last journey on 10th January, 2014. He was only 46 years old. He had lots of contributions especially on understanding of water oxidation mechanism in Photosystem II. Although we waited his manuscript for this special issue, the progress of the disease was so rapid to complete the manuscript. We pay a tribute to the memory of Warwick (see Figs. 1, 2, 3, 4, 5). 


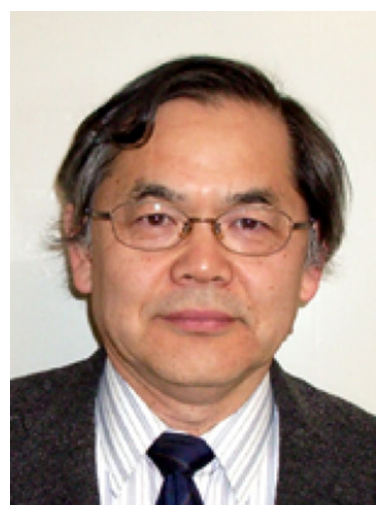

Mamoru Nango

Guest Editor

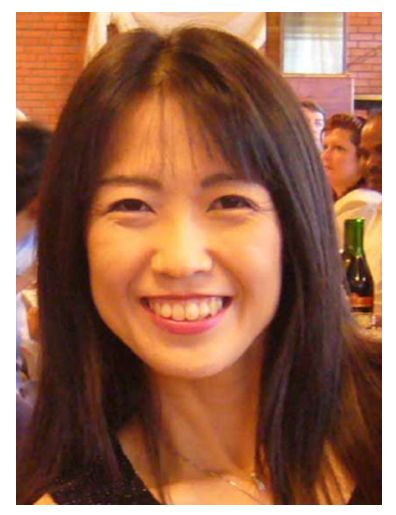

Miwa Sugiura

Guest Editor 


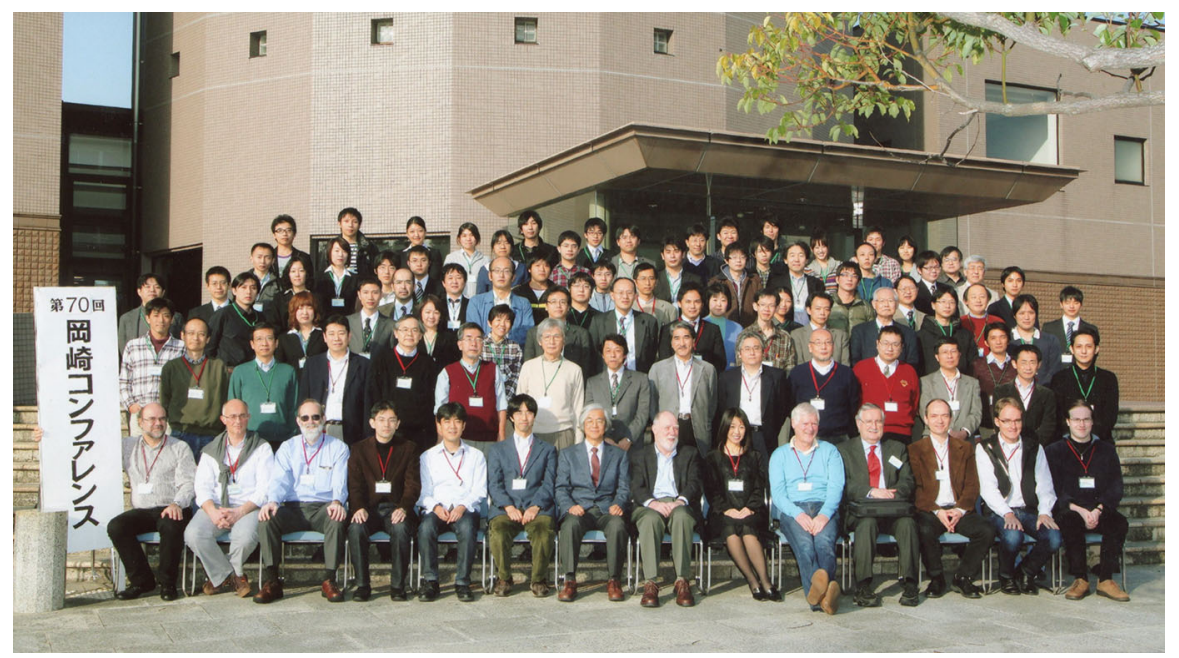

Fig. 1 A group photograph of the 70th Okazaki Conference "Molecular mechanism of photosynthetic energy conversion: the present research and future prospects"
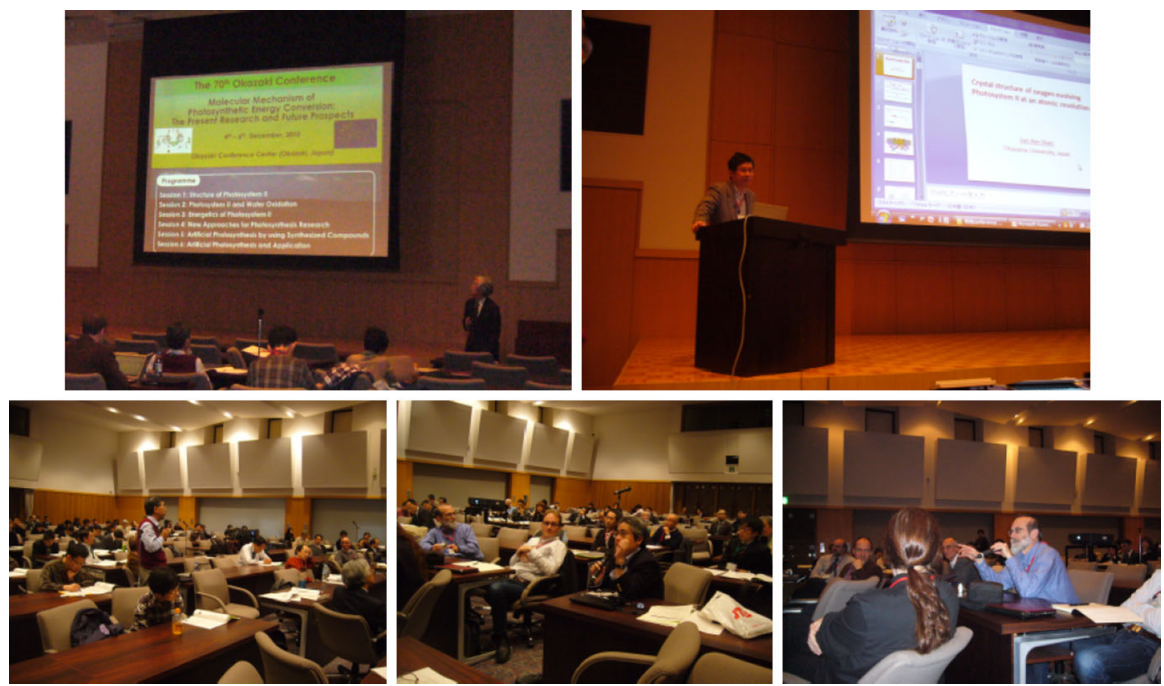

Fig. 2 Talks and discussions. Top left Opening address by Professor Iwao Ohmine (Director-general of Institute for Molecular Science). Top right Professor Jian-Ren Shen (Okayama University) who revealed the X-ray crystal structure of Photosystem II complex at 1.9- $\AA$ resolution 

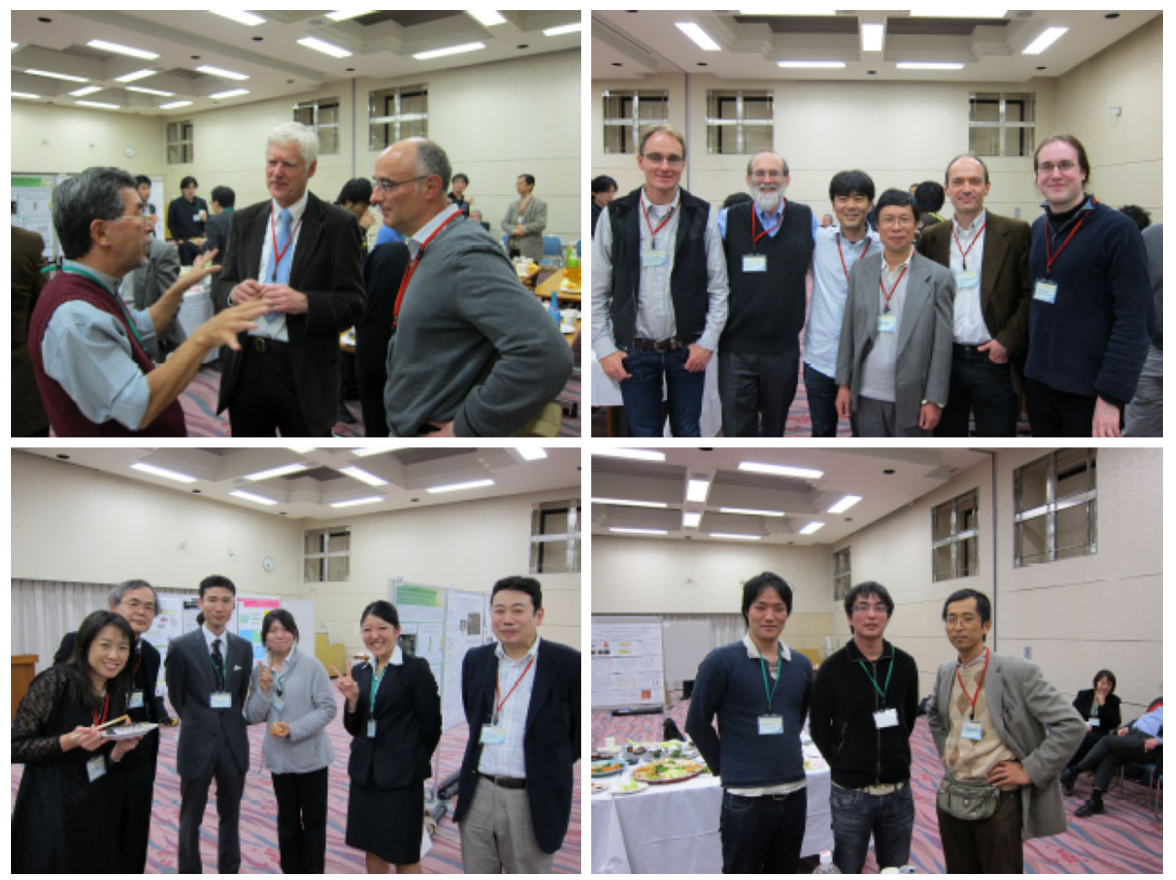

Fig. 3 Some participants at the banquet
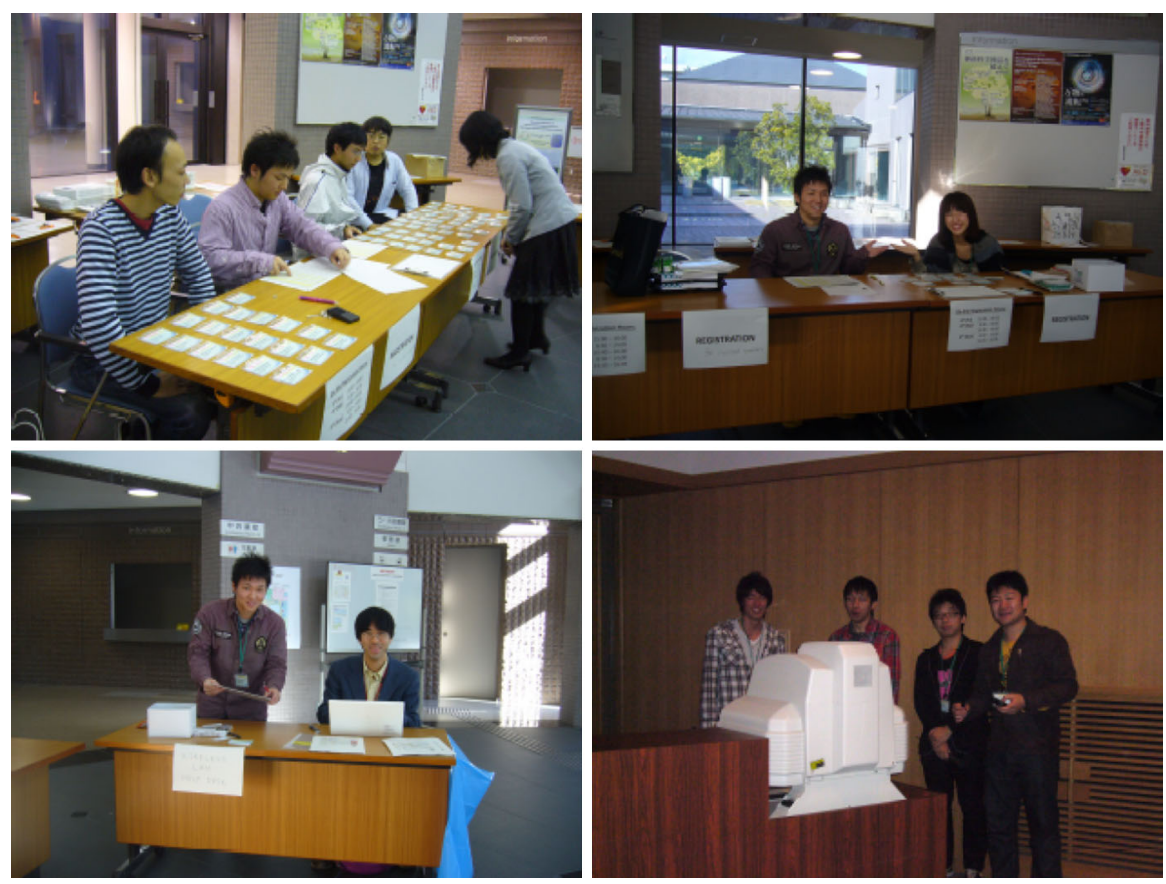

Fig. 4 Volunteer students at the registration desk and in the conference room 

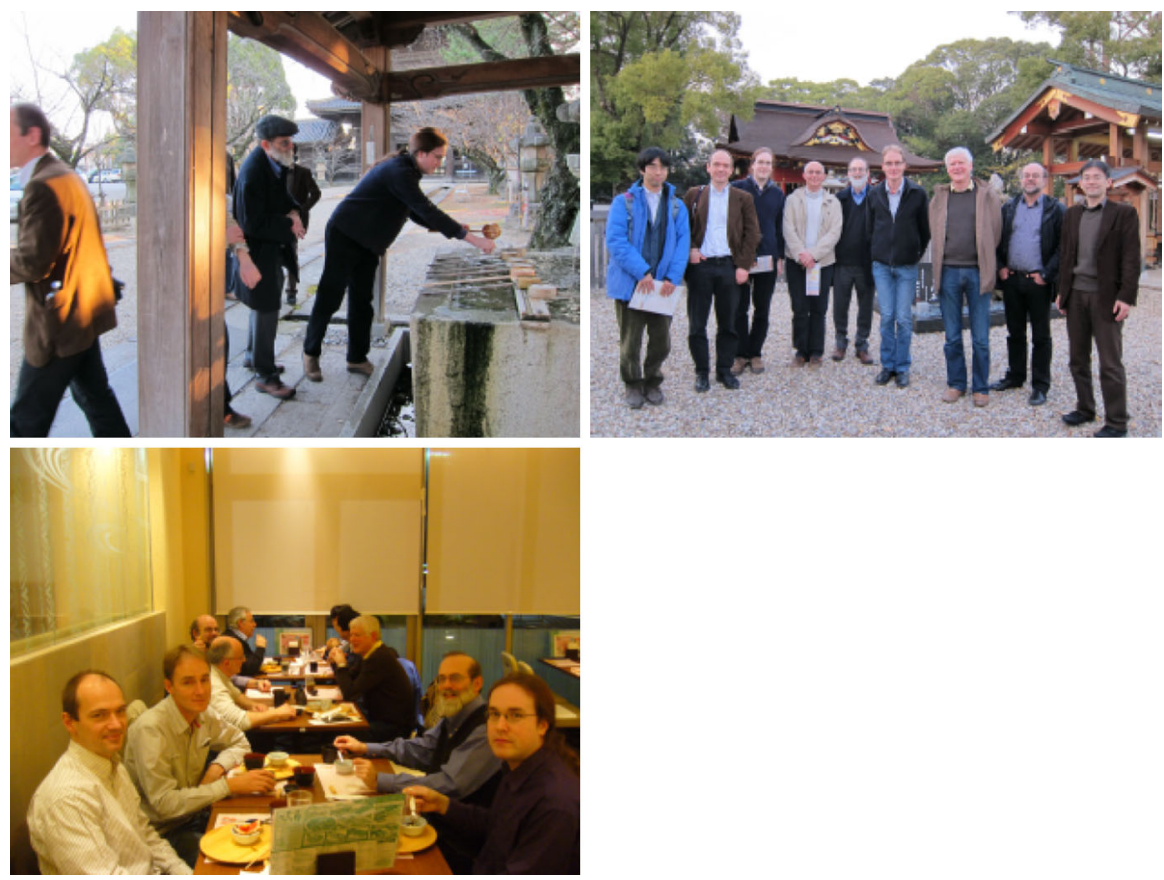

Fig. 5 Visit to Okazaki Park 